(REVIEW ARTICLE)

\title{
The evolving burden of asthma and contemporary advances in management: Implications for clinical practice in Southern Africa
}

\author{
Andrew Kiboneka * \\ Department of Pediatrics, Hage Geingob Campus, School of Medicine Faculty of Health Sciences, University of Namibia.
}

Publication history: Received on 19 August 2020; revised on 15 November 2020; accepted on 21 November 2020

Article DOI: https://doi.org/10.30574/wjarr.2020.8.3.0315

\begin{abstract}
Asthma is a rising significant global public health burden especially in the developing countries. The annual prevalence of severe asthma episodes is estimated from $1 \%$ to $21 \%$ for adults and over $20 \%$ for children aged $6-7$ years. The prevalence of asthma varies widely around the world, ranging from $0.2 \%$ to $21.0 \%$ in adults and from $2.8 \%$ to $37.6 \%$ in 6- to 7-year-old children. The International Study of Asthma and Allergies in Children (ISAAC) reports a significant increase in the global prevalence of asthmatic episodes among children. $t$ was estimated that more than 339 million people had Asthma globally in 2016. It is a common disease among children. The common disease asthma is probably not a single disease, but rather a complex of multiple, separate syndromes that overlap. Most asthma-related deaths occur in low- and lower-middle income countries. According to the World Health Organization (WHO) estimates, there were 417,918 deaths due to asthma at the global level and 24.8 million DALYS attributable to Asthma in in 2016. The WHO has estimated that the economic costs associated with asthma have exceeded those of TB and HIV/AIDS combined, and the Global Initiative for Asthma Program forecasted the number of asthma patients to grow globally to greater than 400 million by the year 2025. Since its first description by Hippocrates, asthma remains a treatable yet incurable disease. It is now clear that asthma is a complex syndrome with variable severity, natural history and response to treatment
\end{abstract}

In Namibia a prevalence of Asthma of $11.2 \%$ has been reported in adult populations. The increase in asthmatic episodes, morbidity and mortality among populations in Africa, Latin America and parts of Asia is a rising public health concern.

The development of novel asthma phenotyping \& endo typing plus better classification of patients using machine learning and big data have markedly improved asthma treatment outcomes in both children and Adults. Several research groups have developed cluster analyses of phenotypes in severe asthma. These clusters support the importance of disease heterogeneity in asthma and suggest differences in pathophysiologic mechanisms that define these clusters. Precision medicine is "an emerging approach for disease treatment and prevention that takes into account individual variability in genes, environment, and lifestyle for each person.

Keywords: Asthma Syndrome; TH2 High, TH2 Low; Phenotypes; Endo-Types; Machine Learning; Asthma Signatures; Microarray Analysis; Biological Therapy; Thermo-Broncho Plasty. Precision Medicine.

\section{Introduction}

Asthma is a common, chronic inflammatory disease of the airways [1,2]. Airway inflammation is associated with airway hyper-reactivity or bronchial hyper-responsiveness (BHR), which is defined as the inherent tendency of the airways to narrow in response to various stimuli (eg, environmental allergens and irritants.

\footnotetext{
${ }^{*}$ Corresponding author: Andrew Kiboneka

Department of Pediatrics, Hage Geingob Campus, School of Medicine Faculty of Health Sciences, University of Namibia. 
Asthma affects an estimated 339 million individuals worldwide. The prevalence of asthma is increasing, especially in children. Annually, the World Health Organization (WHO) has estimated that 15 million disability-adjusted life-years are lost and 250,000 asthma deaths are reported worldwide. Approximately 500,000 annual hospitalizations (34.6\% in individuals aged $18 \mathrm{y}$ or younger) are due to asthma.

The cost of illness related to asthma is around $\$ 6.2$ billion. Each year, an estimated 1.81 million people (47.8\% in individuals aged $18 \mathrm{y}$ or younger) require treatment in the emergency department. Among children and adolescents aged 5-17 years, asthma accounts for a loss of 10 million school days and costs caretakers $\$ 726.1$ million because of work absence

Severe asthma has recently been defined by the World Health Organization as 'uncontrolled asthma which can result in risk of frequent severe exacerbations (or death) and/or adverse reactions to medications and/or chronic morbidity (including impaired lung function or reduced lung growth in children)' [2].

Compared with individuals with mild disease, severe asthmatics often have late disease onset, decreased atopy, elevated sputum neutrophilia and impaired pulmonary function [3]. Focusing on asthma as a syndrome composed of subtypes means that researchers can better focus on treatment options and guidelines for each subtype, rather than just treating all cases of asthma the same.

\section{Materials and methods}

The search strategy included all articles on PuB Med Search Engine, published in English between January 2010 and June 2020.The search terms were advances in case definition, classification (i.e. phenotypes and endo-types) and heterogeneity of asthma. In addition, we reviewed immune cells, biomarkers, and the role of the epithelium plus biological agents in the management of severe asthma in children, adolescents and adults.

\section{Results}

When the diagnosis of asthma is confirmed and comorbidities addressed, severe asthma is defined as asthma that requires treatment with high dose inhaled corticosteroids plus a second controller and/or systemic corticosteroids to prevent it from becoming "uncontrolled" or that remains "uncontrolled" despite this therapy. (GINA Step four). Asthma is a clinical syndrome of chronic airway inflammation characterized by recurrent, reversible, airway obstruction. Airway inflammation also leads to airway hyper reactivity, which causes airways to narrow in response to various stimuli [4]. Severe asthma is a heterogeneous disease characterized by variable airflow obstruction with cough, dyspnea, and wheezing. Such patients are at risk of exacerbations, which may lead to hospitalization, and in rare circumstances, death. Over the past decades, the prevalence of severe asthma has been estimated to be around $5 \%$ to $10 \%$ of the total asthmatic population. Majority of patients with asthma can be treated effectively with the currently available medications. However, a proportion of patients labeled as "severe asthma" remain a challenge for the treating clinician. Recently, monoclonal antibodies targeting interleukin-4 receptor, interleukin-5, and interleukin-13 have demonstrated efficacy by reducing asthma exacerbations when administered in addition to standard therapies in severe asthma [5].

\section{Discussion}

Asthma is a chronic obstructive disease involving the lower airways that carry air in and out of the lungs. These airways are inflamed in people with asthma. The inflammation makes the airways very sensitive, and the tubes often react to allergens or irritations. With the proper diagnosis, medication and an asthma management plan, symptoms can be controlled $[4,5]$

An accurate assessment of asthma severity is essential to predict future risk and impairment and to guide asthma management. The National Asthma Education and Prevention Program and Global Initiative for Asthma guidelines divide asthma severity based on lung function (FEV1), daytime and nocturnal symptoms, and frequency of rescue bronchodilator use $[4,5]$. There is increasing evidence, however, that this approach does not reflect the heterogeneous characteristics of this disease that are observed in populations with asthma. Identification of heterogeneity and classification of asthma by phenotypes provides a foundation from which to understand disease causality and ultimately to develop management approaches that lead to improved asthma control while avoiding adverse effects and decreasing the risk of serious asthma outcomes (e.g., exacerbations and loss of pulmonary function). 
Asthma heterogeneity and complex therapeutic management strategies are more easily recognized in severe asthma, where patients have diverse symptom profiles and altered responses to medications [4, 5]. The, National Heart Lung and Blood Institute-sponsored Severe Asthma Research Program (SARP) is a US multi- center study comprising both children and adults with persistent asthma. The Severe Asthma Research Program cohort includes subjects both adults and children with persistent asthma who have undergone detailed phenotypic characterization. Patients with severe asthma require treatment with high-dose inhaled corticosteroids plus a second controller to maintain symptom control or have uncontrolled asthma despite therapy.

Although these patients are estimated to represent $5 \%$ to $10 \%$ of the total asthma population in the United States, they have frequent exacerbations, impaired quality of life, and account for more than half of the annual costs of asthma. From 2001 to 2012 the National Institutes of Health/National Heart Lung Blood Institute (NIH/NHLBI) supported the Severe Asthma.

Research Program (SARP) I and II cohorts to study mechanisms differentiating severe from non-severe asthma. SARP investigators characterized severe asthma as a heterogeneous syndrome with diverse molecular, biochemical, and cellular inflammatory features and structure-function abnormalities.

Adults and children with severe asthma were further categorized by unbiased statistical methods into clusters based on distinguishing clinical features. These studies have not been done in Sub-Sahara Africa.

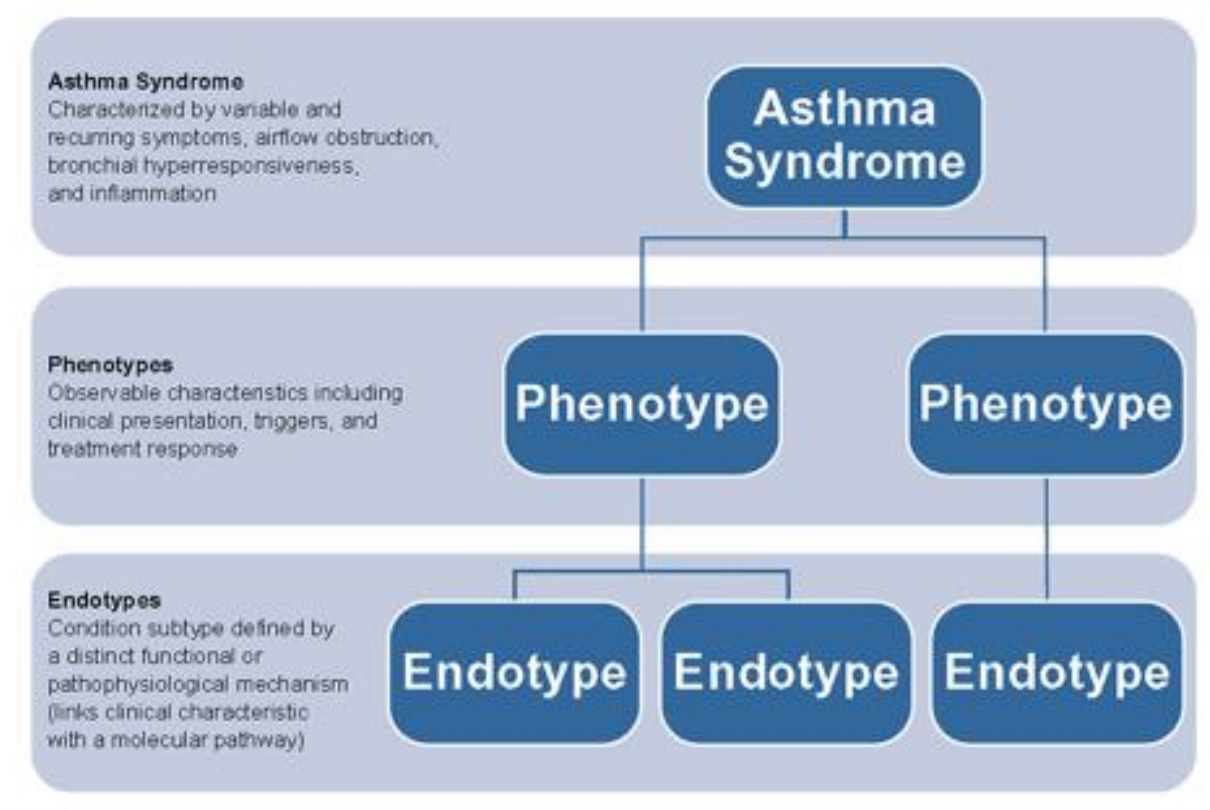

Figure 1 Asthma syndrome, phenotypes and endotypes .Ref: Egypt J Pediatr Allergy Immunol 2016; 14(1):3-14.

Research performed over the past one to two decades has sought to better understand the heterogeneous clinical nature of asthma. Whereas older attempts at phenotyping asthma emphasized the duality of allergic vs. non-allergic asthma, more recent non-biased analyses have attempted to cluster patients by a multitude of possible features, including age of onset, atopy, severity of airways obstruction, and requirement for medication. Examples of these phenotypes include early-onset mild allergic asthma, later-onset asthma associated with obesity, and severe non-atopic asthma with frequent exacerbations. The elucidation of asthma phenotypes has been further refined by including information regarding pathophysiologic mechanisms present in different groups. These groups, called endo-types, include examples such as aspirin-exacerbated respiratory disease and allergic bronchopulmonary mycosis.

A phenotype covers the clinically relevant properties of the disease, but does not show the direct relationship to disease etiology and pathophysiology. Different patho-genetic mechanisms might cause similar asthma symptoms and might be operant in a certain phenotype. These putative mechanisms are addressed by the term 'endotype'.

Classification of asthma based on endo-types provides advantages for epidemiological, genetic, and drug-related studies. A successful definition of endo-types should link key pathogenic mechanisms with the asthma phenotype. Thus, 
the identification of corresponding molecular biomarkers for individual pathogenic-mechanism underlying phenotypes or subgroups within a phenotype is important.

The term asthma encompasses a disease spectrum with mild to very severe disease phenotypes whose traditional common characteristic is reversible airflow limitation. Unlike milder disease, severe asthma is poorly controlled by the current standard of care. Ongoing studies using advanced molecular and immunological tools along with improved clinical classification show that severe asthma does not identify a specific patient phenotype, but rather includes patients with constant medical needs, whose patho-biologic and clinical characteristics vary widely. Accordingly, in recent clinical trials, therapies guided by specific patient characteristics have had better outcomes than previous therapies directed to any subject with a diagnosis of severe asthma.

The term "eosinophilic" asthma (EA) generally refers to the clinical inflammatory phenotype of asthma wherein a significant number of sputum, airway, and/or blood eosinophils are present. Conversely, individuals with "noneosinophilic"asthma (NEA) may still demonstrate low numbers of eosinophils, but the dominant inflammatory cell type may include neutrophils, mixed granulocyte inflammatory cells, or very few inflammatory cells, termed paucigranulocytic inflammation [6].

\subsection{The immune system \& cells in asthma}

Generally, immunity is divided into 2 components, the innate and adaptive immune system. The adaptive immune system is further divided into the cellular and humoral immune systems. The complement system functions in both innate and adaptive immunity. The innate immune system is comprised of proteins and cells, including monocytes, neutrophils, macrophages, and natural killer cells. The cellular and humoral immune systems is composed of T- and Blymphocytes, respectively. However, this is a simplistic approach to the immune system because there is cross-talk between components of the various systems and defects can occur that affect several aspects of immunity simultaneously.

Asthma is an inflammatory disease of the airway wall that leads to bronchial smooth muscle hyper-reactivity and airway obstruction, caused by inflammation, goblet cell metaplasia, and airway wall remodeling. In response to allergen presentation by airway DCs, T-helper lymphocytes of the adaptive immune system control many aspects of the disease through secretion of IL-4, IL-5, IL-13, IL-17, and IL-22, and these are counterbalanced by cytokines produced by Treg cells. The recognition that asthma is primarily an inflammatory disorder of the airways associated with T helper type 2 (TH2) cell-dependent IgE production and recruitment of mast cells and eosinophils has provided the rationale for disease control using inhaled corticosteroids and other anti-inflammatory drugs. Recent advances in our understanding of the sentinel role played by innate immunity provides new targets for disease prevention and treatment. These include pathways of innate stimulation by environmental or endogenous pathogen-associated molecular patterns (PAMPs) and danger-associated molecular patterns (DAMPs) to influence the activation and trafficking of DCs, innate sources of cytokines, and the identification of new T cell subsets and lymphoid cells.

Many cells of the innate immune system such as mast cells, basophils, neutrophils, eosinophils, and innate lymphoid cells also play an important role in asthma immmuno-pathogenesis.

Much of the focus in asthma research in the past has been on adaptive, antigen-dependent immune responses. Recent work suggests that the innate, non-antigen-dependent immune system plays a critical role in asthma pathogenesis.

Thymic stromal lymphopoietin (TSLP), interleukin-25 (IL-25), and IL-33 are important initiators of type 2-associated mucosal inflammation and Immunity. Innate cells contribute to the development of asthma clinical manifestations. Epithelial cell-DC (Dendrtitic Cell) crosstalk is crucial for asthma development [7, 8, and 9]. Epithelium-derived cytokines contribute to ILC2 responses. (10) Traditionally, asthma is an inflammatory disease of the airways associated with a T helper (Th)2 response. Such a response in the lungs requires complex interactions between innate cells and structural cells. . Patients with allergic asthma have eosinophilic inflammation in the lung, in parallel with increased mediators associated with an adaptive, allergen-dependent response (e.g., interleukin [IL] 4, IL-5, and IL-13) of the T helper cell (Th)2 rather than Th1subset (e.g., interferon [IFN]- $\gamma$ ) , as well as elevated serum immunoglobulin (Ig)E. Recent attention has also focused on the Th17 subset that mediates neutrophilic inflammation and plays a role in asthma immiuno-pathogenesis.

Dendritic cells (DCs) are pivotal during sensitization to allergens but clearly require epithelium-derived interactions to become activated. Epithelial cells also contribute to the activation and the survival of mast cells (MCs), basophils, and eosinophils and group 2 innate lymphoid cells (ILC2s). In turn, these innate cells can activate DCs to sustain Th2 
immunity and orchestrate (produce) TH2 immunity. The pathophysiology of asthma is complex and involves airway inflammation, intermittent airflow obstruction, and bronchial hyper responsiveness. Antigen presentation by the dendritic cell with the lymphocyte and cytokine response leading to airway inflammation and asthma symptoms .Although the mechanisms driving EA are becoming clearer, by contrast much less attention has been given to the underlying mechanisms that drive NEA, leading to a lack of available therapies for this patient population.

Although "phenotype" refers to the observable characteristics of disease in an individual, by "endotypes" we mean the specific biological mechanism that causes those observed properties of any given phenotype. Understanding the manifold mechanisms or endo-types contributing to EA and NEA and the resulting clinical phenotypes derived from these endo-types has lead to novel treatment options and provide an opportunity for more precise and tailored treatments for asthma both in children \& adults.

\subsection{The airway epithelium in asthma}

The airway epithelium is considered an essential controller of inflammatory, immune and regenerative responses to allergens, viruses and environmental pollutants that contribute to asthma pathogenesis. Epithelial cells express pattern recognition receptors that detect environmental stimuli and secrete endogenous danger signals, thereby activating dendritic cells and bridging innate and adaptive immunity. .

Antigen presentation by the dendritic cell with the lymphocyte and cytokine response leading to airway inflammation and asthma symptoms. Some of the principal cells identified in airway inflammation include mast cells, eosinophils, epithelial cells, macrophages, and activated T lymphocytes.

The airway epithelium represents a sealed, self-cleaning barrier, that prohibits penetration of inhaled allergens, pathogens, and other noxious agents into the body. This barrier is covered with mucus that further contains antimicrobial peptides and antibodies that are either produced or specifically transported by the airway epithelium in order to trap these particles and to remove them from the body by a process called mucociliary clearance. Once this first line of defense of the lung is overcome, airway epithelial cells are the first cells to get in contact with pathogens, to be damaged or infected. Therefore, these cells release a plethora of chemokines and cytokines that not only induce an acute inflammatory reaction but also have an impact on the alignment of the following immune reaction. In case of asthma, all these functions are impaired by the already existing allergic immune response that per se weakens the barrier integrity and self-cleaning abilities of the airway epithelium making it more vulnerable to penetration of allergens as well as of infection by bacteria and viruses. Recent studies indicate that the history of allergy- and pathogen-derived insults can leave some kind of memory in these cells that can be described as imprinting or trained immunity. Thus, the airway epithelium is in the center of processes that lead to formation, progression and acute exacerbation of asthma.

\subsection{Asthma signatures}

A compendium of global gene expression measurements from DNA microarray analysis of immune cells identifies gene expression signatures defining various lineages, differentiation stages, and signaling pathways [11, 12].

The observation that subsets of asthmatic patients exist who continue to have symptoms despite maximal medical therapy has motivated the search for distinct asthma subgroups with putative differences in disease mechanisms. Moore et all [5] demonstrated the presence of 5 distinct phenotypic clusters among adult asthmatic patients, and Fitzpatrick et al [5] performed a parallel analysis with analogous findings among patients with childhood asthma.

An important implication of recent advances in our understanding of asthma phenotypes is that we can use these clusters to uncover associated differences in patho-genetic mechanisms and thus have the potential to identify new therapeutic targets with increased treatment specificity and new molecular biomarkers for improved clinical detection. Characteristic features of atopic asthma are circulating specific IgE antibodies, positive skin tests to common allergens, and infiltration of the bronchial mucosa with eosinophils and Th2 cells. The resulting pulmonary inflammation leads to bronchoconstriction, airway hyper-responsiveness, and ultimately to airway remodeling.

\subsection{Micro-array analysis}

A DNA microarray (also commonly known as DNA chip or biochip) is a collection of microscopic DNA spots attached to a solid surface. Scientists use DNA microarrays to measure the expression levels of large numbers of genes simultaneously or to genotype multiple regions of a genome. Each DNA spot contains picomoles (10-12 moles) of a specific DNA sequence known as probes (or reporters or oligos). DNA microarrays can be used to detect DNA (as in comparative genomic hybridization), or detect RNA (most commonly as cDNA after reverse transcription) that may or 
may not be translated into proteins. The process of measuring gene expression via cDNA is called expression analysis or expression profiling.

Applications of microarray analysis includes study of Asthma: Identifying single nucleotide polymorphism(SNP) among alleles within or between populations. Several applications of microarrays make use of SNP detection, including genotyping, forensic analysis, measuring predisposition to disease, identifying drug-candidates, evaluating germline mutations in individuals or somatic mutations in cancers, assessing loss of heterozygosity, or genetic linkage analysis.

\subsection{Machine learning (Big data)}

Machine learning (ML) is the scientific study of algorithms and statistical models that computer systems use to effectively perform a specific task without using explicit instructions, relying on patterns and inference instead. It is seen as a subset of artificial intelligence. Arthur Samuel, an American pioneer in the field of computer gaming and artificial intelligence, coined the term "Machine Learning" in 1959 while at IBM(International Business Machines Corporation). As a scientific endeavor, machine learning grew out of the quest for artificial intelligence(Robots).

\subsection{Asthma classification}

Asthma can be broadly classified as eosinophilic or non-eosinophilic on the basis of airway or peripheral blood cellular profiles, with approximately half of individuals with asthma falling into each category. Sputum cellular profiles are believed to directly reflect lung inflammation.

And therefore, are the preferred method used in asthma research to determine EA. Sputum eosinophils should be reported as a percentage of total cells from either a whole expectorate or sputum plug. Sputum eosinophil levels of greater than 2 to $3 \%$ have been used to define EA. However, technical requirements for sputum processing and cell counting may limit the feasibility of using sputum eosinophil counts in all clinical centers especially in Sub-Saharan Africa \& other developing countries.

\subsection{New biologics for severe asthma}

The title of a well-attended symposium initiated by the editor of the New England Journal of Medicine at the recent meeting of the European Respiratory Society in Paris. Physicians treating patients with asthma need guidance on how to best manage severe disease and what drugs are the most appropriate in chronic care. Although this generally applies to all therapeutic measures, the further development of biologics requires a proper, patient-centered approach that is economically responsible.

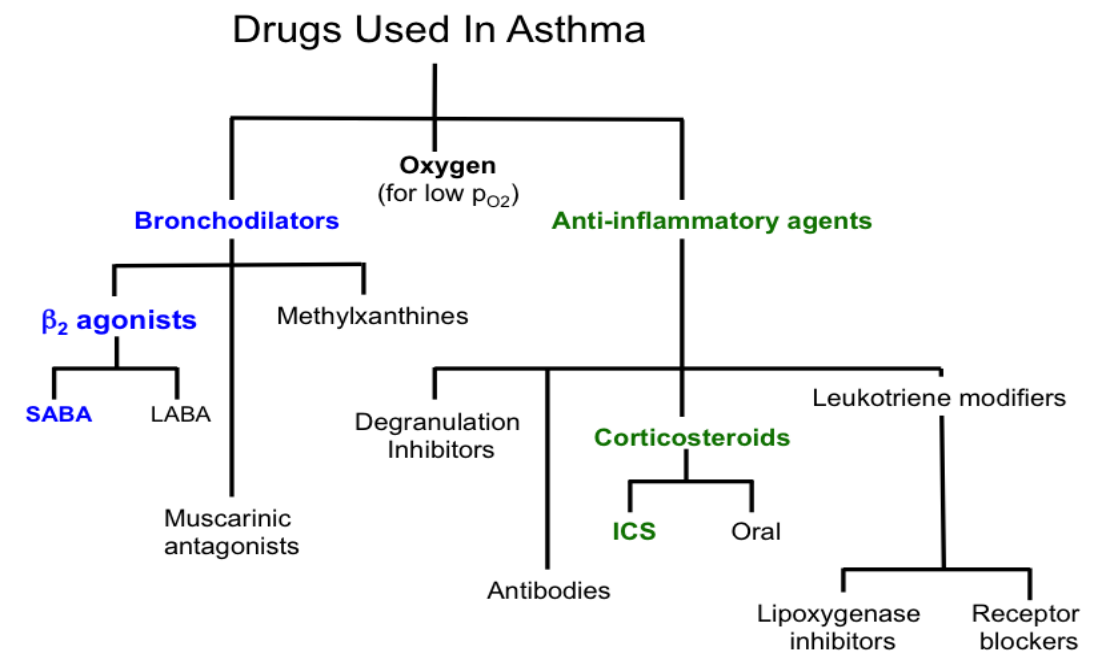

Figure 2 Drugs used in the treatment of asthma.

This discussion is not new, and since the introduction of antibodies targeting IgE, investigators have searched for a clinically applicable rule to identify patients who would benefit from this treatment, and for indicators to estimate how long such a costly treatment should last. One approach that has been used, the Global Evaluation of Effectiveness scale largely relies on subjective impressions of treatment success by the physician and the patient. 


\subsection{Airway epithelial cells}

The $2^{21}$ to $2^{23}$ branches of the adult human airways are covered with a continuous epithelial sheet comprising less than $1 \%$ of the total respiratory epithelial surface. The airway epithelium is pseudostratified in the large airways, becoming columnar and cuboidal in the small airways. The major cell types are ciliated, columnar, undifferentiated, secretory and basal cells. Epithelial damage is a characteristic feature of asthma. The epithelium is not merely a passive barrier but can generate a range of mediators that may play a role in the inflammatory and remodeling responses that occur in the lungs in asthma. Clara cells, another specialized epithelial type of the terminal airways is found in broncheo- alvelolar transition regions.

Development of allergic asthma is a complex process involving immune, neuronal and tissue cells. In the lung, Clara cells represent a major part of the "immunomodulatory barrier" of the airway epithelium. Clara cells augment the allergic immune response in the lung.

\subsection{The use of biomarkers to endo-type patients with severe asthma}

Biomarkers are a major step forward, and they allow us to personalize treatment strategies. Specific biomarkers can play a very important role in helping us to determine when a patient is expressing type 2 asthma-and, as a consequence, which treatments may be most effective.

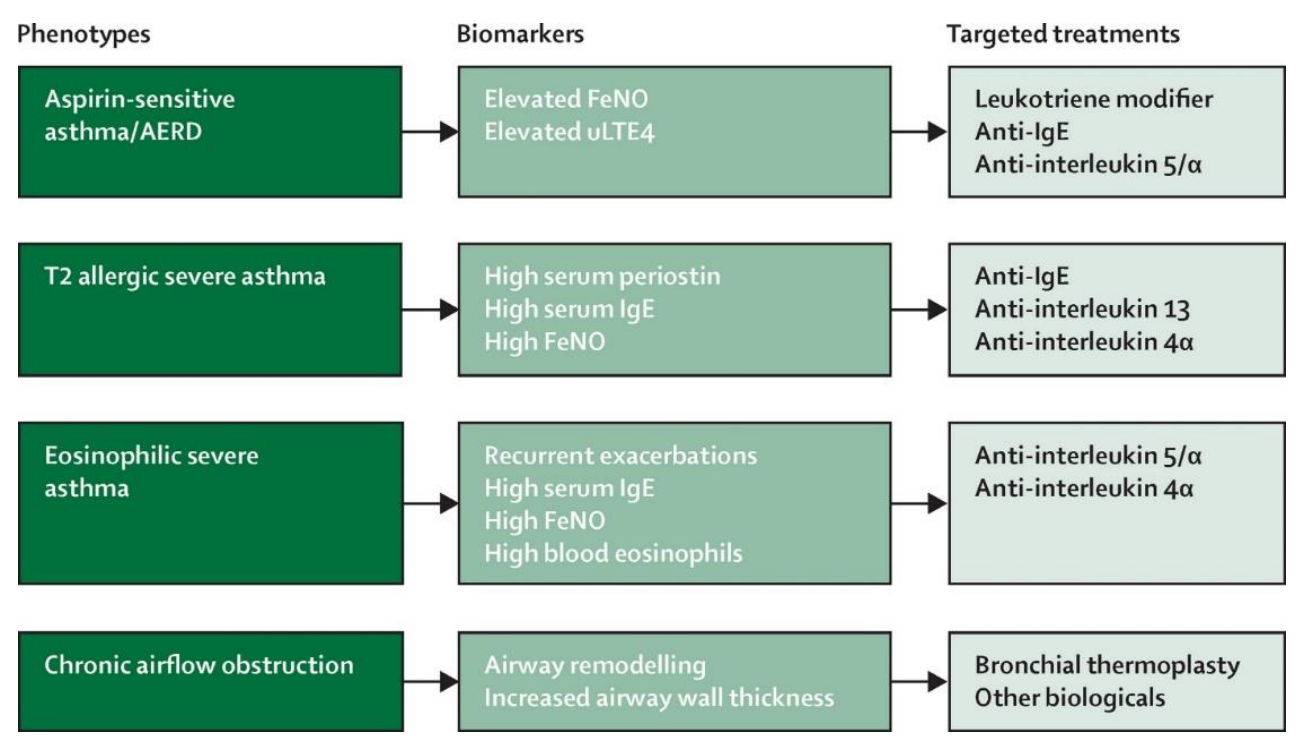

Figure 3 Asthma phenotypes asoociated endotypes and targeted treatments

The phenotypes in type 2 inflammation have two major groupings-early childhood-onset disease, which is allergic in nature, or later adult-onset, which is associated with nasal polyposis and sinusitis frequently. However, both are associated with the presence of biomarkers-exhaled nitric oxide, eosinophils, IgE, and in experimental situations, periostin.

Non-type 2 asthma is just as important as type 2, but the mechanisms associated with it are not clearly understood. Furthermore, there are no biomarkers, other than the possibility of neutrophilia in the airway, to tell us about the presence of this disease. There are currently a number of asthma biomarkers and methods to detect them. Dupilumab, which is an anti-IL-4 alpha receptor antibody which also affects IL-13, has been shown to be most effective with patients having eosinophils at 300 cells $/ \mu \mathrm{L}$ and to some degree with periostin.

If we look at those biologics that target IL-5, we see different levels of eosinophils as far as recommended levels for the use of these products. Whereas with benralizumab, there is some evidence to suggest that exhaled nitric oxide 50 parts per billion may be helpful in this regard. In considering the anti-IL-5 pathways, there needs to be a certain level of eosinophilia before these products are going to show efficacy. With IgE, patients who express a T2 pattern are more likely to respond. The prescribing of omalizumab is going to be dependent upon their IgE level. 
Importantly, when exhaled nitric oxide is elevated, particularly in the non-treated situation, there's airway inflammation present, and there are a number of advantages in the utility of exhaled nitric oxide in the diagnosis of asthma.

\subsection{Major efforts to further unravel the mechanisms of severe asthma have been undertaken and have delivered a wide range of drug targets, such as}

- IL-5,

- The transcription factor GATA-3,

- L-4, IL-13, TSLP (thymic stromal lymphopoietin).

- The prostaglandin DP2 (CRTH2) : receptor

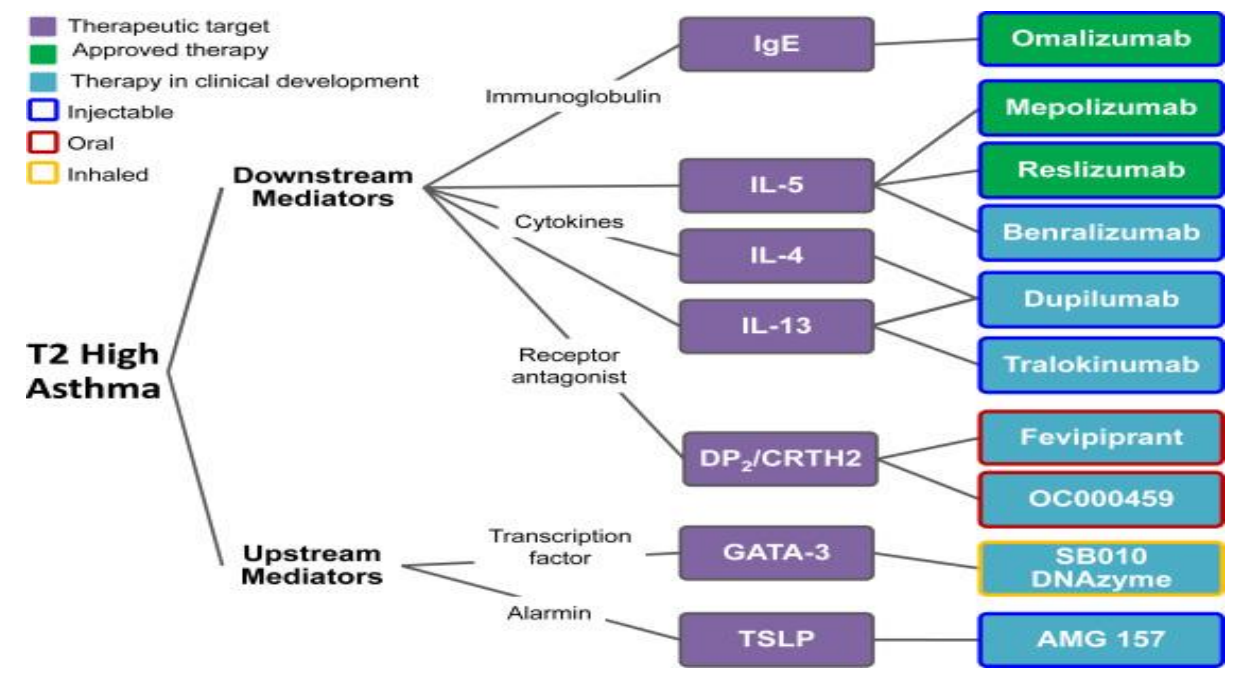

Figure 4. TH2 high asthma= Th2 cells and/or their secreted effector molecules mediate the immune response to allergens and are triggered by exposure to specific allergens leading to allergic asthma. Source: [14].

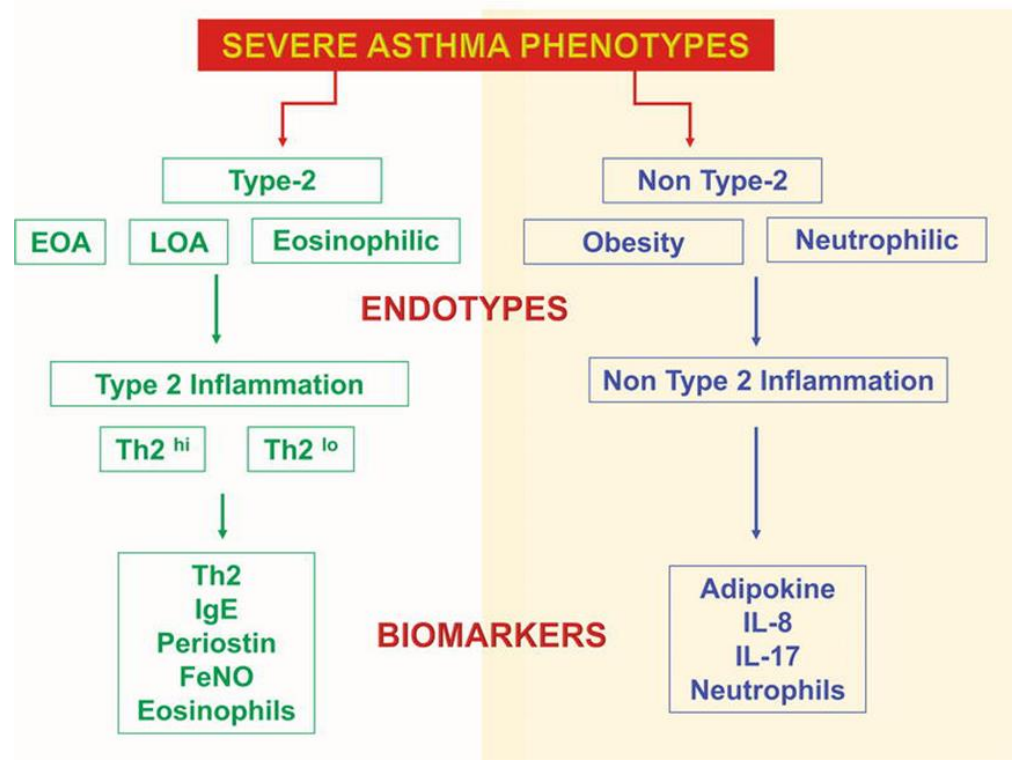

Figure 5 Phenotypes, endotypes, and biomarkers in severe asthma. 
Asthma is divided into phenotypes: type 2 inflammation and non-type 2 inflammation. Type 2 phenotype: early onset asthma (EOA), late onset asthma (LOA), and eosinophilic asthma; biomarkers: Th2 cytokines, IgE, Periostin, FeNO (fraction of nitric oxide expired), and eosinophilia. Non-type 2 phenotypes: asthma associated with obesity and neutrophilic asthma; biomarkers: adipokine, IL-8 or IL-17, and neutrophilia.Ref: Severe Asthma: Updated Therapy Approach Based on Phenotype and Biomarker. Source: [15].

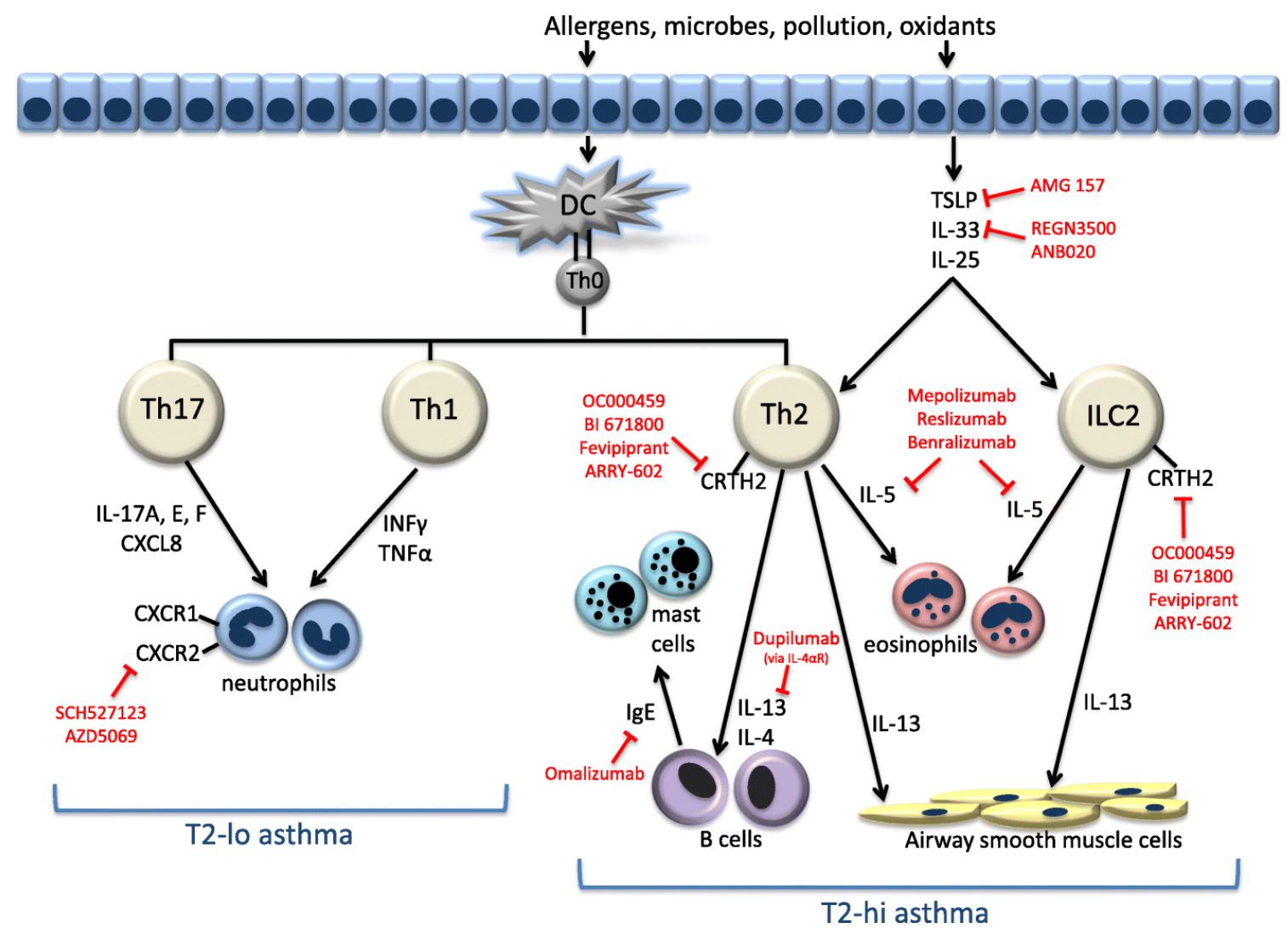

Figure 6 Allergens and immunopathogenesis of asthma

Among these, the cytokine IL-5 and its receptor are the most advanced and have yielded a number of drugs that are now available for clinical use. Although there are different paradigms for establishing relevant phenotypes, asthma can broadly be separated into two categories: eosinophilic (T-helper 2 [Th2]) and non-eosinophilic (non-Th2) [4]. Each category has a different underlying immune-biology, and recently approved medications for asthma all target the eosinophilic/Th2 type of disease. For patients with eosinophilic/Th2 asthma, the disease is typically exacerbated by inhaled "triggers."

Triggers include allergens, pollutants, and infections that make contact with the airway epithelium and induce an inflammatory cascade. This cascade includes multiple cytokines, or cellular messengers, that help coordinate an immune response to a given trigger. Cytokines, such as interleukins (ILs), interact with cells and immunoglobulins (Igs) to activate eosinophils, mast cells, and leukotrienes, and stimulate mucus and histamine release. The end result for the patient is bronchoconstriction; chest congestion; and cough, dyspnea, and wheezing. Recently developed monoclonal antibodies, also referred to as "biologics," target different levels of the eosinophilic/Th2 asthmatic response.Biologics are finding their way into clinical practice and owing to their efficacy will be used globally and not only in specialized centers in the developed world.

\subsection{Childhood asthma: Advances using machine learning}

A paradigm shift brought by the recognition that childhood asthma is an aggregated diagnosis which comprises of several different endo-types underpinned by different pathophysiology, coupled with advances in understanding potentially important causal mechanisms, offers a real opportunity for a step change to reduce the burden of the disease on individual children, families and society. Data-driven methodologies facilitate the discovery of "hidden" structures 
within "big healthcare data" to help generate new hypotheses. These findings can be translated into clinical practice by linking discovered "phenotypes" to specific mechanisms and clinical presentations.

Severe asthma often has an eosinophilic phenotype with elevated blood and lung eosinophil levels. IL-5 is the major cytokine responsible for the growth, differentiation, recruitment, activation, and survival of eosinophils. Mepolizumab and reslizumab, the two previously approved anti-IL- 5 antibodies, reduce the production and survival of eosinophils by binding to IL-5, preventing it from binding to its receptors. Benralizumab binds directly to the IL-5 receptor, preventing IL-5 binding and causing apoptosis of eosinophils and basophils through cell-mediated cytotoxicity. All three of these agents decrease eosinophilic airway inflammation.

\section{Airway remodeling:}

Pathogenic changes that increase airway wall thickness and decrease airway luminal diameter, such as mucous cell metaplasia and mucus hyper-secretion, and hyperplasia of airway epithelial cells and smooth muscle cells, and deposition of collagen and extracellular matrix proteins.

\subsection{FDA approved biological for asthma and their mechanism of action:}

There are five FDA approved monoclonal antibodies that affect the pathways involved in either the allergic or type 2 inflammatory phenotypes of asthma. There is increasing recognition of phenotypic variability in pediatric asthma, providing the opportunity for a more personalized approach to therapy. Increasingly biologic therapies, in particular those targeting the "allergic" (or T helper 2) pathway, are being considered for children with severe asthma The drugs, dosing, their mechanisms of action, and their FDA indications for asthma are summarized in the table below:

Omalizumab is a monoclonal antibody to IgE, which is indicated for the treatment of patients with moderate to severe asthma with the allergic phenotype described above.

Mepolizumab, reslizumab, and benralizumab target the IL-5 pathway either with monoclonal antibodies to IL-5 itself (mepolizumab, reslizumab) or to the IL-5 receptor (benralizumab)

Dupilumab is a monoclonal antibody to the IL-4 receptor alpha, which modulates both the IL-4 and IL-13 pathways..

\section{Bronchoplasty:}

Bronchial thermoplasty: a recentlyintroduced treatment for asthma that uses several bronchoscopic procedures to thermally ablate airway smooth muscle

\section{Conclusion}

There remains a high burden of asthma in Sub-Saharan Africa. Disease burden is the impact of a health problem as measured by financial cost, mortality, morbidity, or other indicators. It is often quantified in terms of quality-adjusted life years (QALYs) or disability-adjusted life years (DALYs), both of which quantify the number of years lost due to disease (YLDs). One DALY can be thought of as one year of healthy life lost, and the overall disease burden can be thought of as a measure of the gap between current health status and the ideal health status (where the individual lives to old age free from disease and disability).

Cconsiderable progress in three major areas: deciphering the genetic roots of the disease, understanding the molecular basis of its heterogeneity, and using this new information to improve the lives of patients with the most severe forms of the disease has been achieved.. At the same time, major issues such as controlling exacerbations and attaining satisfactory adherence to inhaled therapy remain unresolvedas well as wide scale implementation of biomarkers and biologicals. for severe asthma in resource limted/developing countries. Almost 80 years ago, Rackemann first reviewed the evidence suggesting that there were at least two forms of asthma: extrinsic and intrinsic, or approximately T2-high and T2-low asthma if we use today's nomenclature. There is no doubt that we have come along way in our grasp of the molecular basis of T2-high asthma,but still, much more needs to be understood about it, and T2-lowasthma remains essentially a mystery. Asthmatics with a severe form of the disease are frequently refractory to standard medications such as inhaled corticosteroids, underlining the need for new treatments to prevent the occurrence of potentially lifethreatening episodes. A major obstacle in the development of new treatments for severe asthma has been until recently the heterogeneous pathogenesis of the disease, which involves multiple mechanisms and cell types. Since the approval of omalizumab in 2003, the development of biologic asthma therapies has grown at a remarkable pace. With 
approximately 30 drugs currently in clinical trials and dozens more in development, the future of asthma biologic therapies is promising.We heve entered a a new era of the evolving definition of asthma and its management in both children and adults.

\section{Recommendations:}

Assessing the quality of evidence and strength of recommendations. Schematic representation of the grade approach for synthesizing evidence and developing recommendations:

The first requirement for any disease program is ensuring the sensible and accurate translation of evidence arising from clinical trials into public health recommendations and policy. This is a complex process that is best approached in a systematic way. The Grading of Recommendations Assessment, Development and Evaluation (GRADE) working group has developed a commonly used tool to evaluate the certainty of findings arising from a systematic review of the evidence, and a separate tool to Advances in big data analytics have created an opportunity for a step change in unraveling mechanisms underlying the development of complex diseases such as asthma, providing valuable insights that drive better diagnostic decision-making in clinical practice, and opening up paths to individualized treatment plans assist with making recommendations for treatment, diagnosis, or prevention. In brief, the available evidence for each outcome of interest is first assessed for quality, from very low to high, according to the confidence in the evidence. This may be downgraded because of variety of reasons. Once the quality of the evidence has been assessed and considered sufficiently robust to merit consideration for translation, the applicability to the wider population must then be evaluated. A strong recommendation would be appropriate when most patients (or their families) would want the intervention (bio-markers \& biologicals), where the majority of clinicians in Namibia agree that the intervention should be offered, and where the recommendations are acceptable as a public health measure to policy makers. The GRADE framework has been used by the Allergic Rhinitis and its Impact on Asthma group and working groups within the World Allergy Organization.

Biomarkers and biologic agents should be made readily available in Sub Saharan Africa, as they have proved to be cost effective in the treatment of Severe Asthma in both children and Adults elsewhere.

\section{Compliance with ethical standards}

\section{Acknowledgment}

The author is thankful to William Busse. MD. University of Wisconsin, School of Medicine \& Public Health. Winsconsin, USA, Russel Hopp MD. Creighton University, Omaha, Nebraska, USA, and Professor Peter Nyarango, Dean, School of Medicine, University of Namibia, Windhoek, Namibia.

\section{Disclosure of conflict of interest}

The author declares that there has been no conflict of interest .No conflict of interest is thus declared.

\section{References:}

[1] American Academy of Allergy,Asthma and Immunology(AAAAI).

[2] Moore. W.C et al. Clinical Heterogeneity in the Severe Asthma Research Program. Ann Am Thorac Soc Dec 2013; Vol 10, Supplement:pp S118-S124,

[3] Guidelines for the Diagnosis and Management of Asthma (EPR-3). National Heart, Lung, and Blood Institute (NHLBI) of the National Institutes of Health.2007.

[4] Tara F. Carr et al. Eosinophilic and Non-eosinophilic Asthma. Am J Respir Crit Care Med 2018; Vol 19, Iss 1: pp 22-37, Jan 1.

[5] Kevin M. Vannella et al. Combinatorial targeting of TSLP, IL-25, and IL-33 in type 2 cytokine-driven inflammation and fibrosis. www.ScienceTranslationalMedicine.org 4 May 2016; Vol 8 Issue 337 337ra65: Pages 1-13.

[6] Itziar Martinez-Gonzalez et al. Lung ILC2s link innate and adaptive responses in allergic inflammation. Trends in Immunology, March 2015: Vol. 36, No. 3 189: pages 189-196.

[7] Patricia W. Finn et al. Innate Immunity and Asthma. Proc Am Thorac Soc 2009; Vol 6: pp 260-265. 
[8] A.L. Shaffer et al. Signatures of the Immune Response. Immunity, 2001; Vol. 15:375-385, September.

[9] Heung-Woo Park et al. Genetic Signatures of Asthma Exacerbation. Allergy Asthma Immunol Res. 2017 May; 9(3):191-199.

[10] Elissa M. Abrams et al. Current State and Future of Biologic Therapies in the Treatment of Asthma in Child. PEDIATRIC ALLERGY, IMMUNOLOGY, AND PULMONOLOGY. 2018; Volume 31, Number 3,

[11] Emily E. Barsky et al. A Practical Approach to Severe Asthma in Children. Annals. ATS 2018; Volume 15 Number 4| April:Pages 399-408.

[12] Sejal Saglani Et Al. Childhood Asthma: Advances Using Machine Learning And Mechanistic Studies. Ajrccm . Published On 20-December-2018 As 10.1164/Rccm.2018; 10-1956ci

[13] Ceyda Okse et al. Classification of Pediatric Asthma: From Phenotype Discovery to Clinical Practice. Frontiers in Pediatrics | www.frontiersin.org. September 2018; Volume 6:Article 258.

[14] Pepper AN, Renz H, Casale TB, Garn H. Biologic therapy and novel molecular targets of severe asthma. The Journal of Allergy and Clinical Immunology: In Practice. 2017 Jul 1;5(4):909-16.

[15] Piuvezam MR, Ferreira LK, Monteiro TM, Vieira GC, Bezerra-Santos CR. Severe Asthma: Updated Therapy Approach Based on Phenotype and Biomarker. Approach Based on Phenotype and EndotypeAsthma Diagnosis and Management. 2018 Jul 4:125. 